\title{
Primary Effects of New Leishmania major Antigen on Balb/c Mice Spleen
}

Latifynia $A^{1,2^{*}}$, Gharagozlou $\mathbf{M}^{3}$, Khamesipour $A^{4}$, Mohammadi MA $^{4}$ and Khansary $\mathbf{N}^{1}$

${ }^{1}$ Department of Immunology, Faculty of Medicine, Tehran University of Medical Sciences, Tehran, Islamic Republic of Iran

${ }^{2}$ Research Center for Immunodeficiencies, Childeren's Medical Center Hospital, Tehran University of Medical Sciences, Tehran, Islamic Republic of Iran

${ }^{3}$ Departments of Pathobiology, Faculty of Veterinary Medicine, University of Tehran, Tehran, Islamic Republic of Iran

${ }^{4}$ Leprosy and Dermal disease Center, Tehran University of Medical Sciences, Tehran, Islamic Republic of Iran

"Corresponding author: Latifynia Afshineh, Dept of Immunology, Faculty of Medicine, Tehran University of Medical Sciences,Tehran, Islamic Republic of Iran, Tel: 982166439463; E-mail: alatifynia@sina.tums.ac.ir

Received date: 02 July 2014; Accepted date: 22 Sep 2014; Published date: 27 Sep 2014

Copyright: () 2014 Latifynia A, et al. This is an open-access article distributed under the terms of the Creative Commons Attribution License, which permits unrestricted use, distribution, and reproduction in any medium, provided the original author and source are credited.

\begin{abstract}
Background: Cutaneous leishmaniasis is a zoonotic disease transmitted between rodents and canine, mainly induced by phelebotomus sand flies. In Iran, the incidence of this protozoan disease has doubled over the last decade. Regard to its high incidence, in the present study the new antigen preparation was evaluated following challenging with live Leishmania major promastigts.
\end{abstract}

Methods: One hundred and twenty male and female Balb/c mice were randomly divided into four groups as LT, LB, LBT and control groups. Group LT received $100-200 \mu \mathrm{g} / 0.1 \mathrm{ml}$ of the crude cocktail antigen preparation plus alcoholic extract of Teucrium polium as adjuvant, LB received $100-200 \mu \mathrm{g} / 0.1 \mathrm{ml}$ of the crude cocktail antigen preparation plus BCG as adjuvant, LBT received $100-200 \mu \mathrm{g} / 0.1 \mathrm{ml}$ of this antigen preparation plus alcoholic extract of Teucrium polium and BCG as adjuvants and control group. Groups LT, LB and LBT were injected subcutaneously with the antigen preparation and they were boosted using the same doses with two weeks intervals later. A week after the last booster, all animals was challenged with live Leishmania major promastigotes. The animals survived, were euthanized, their spleen was removed, and 4-5 microns paraffin sections were prepared and stained with Harris Hematoxylin and Eosine method. The expansion rates of the spleen white pulp size were evaluated using a light microscope with eye-piece graticule.

Results \& Conclusion: Statistically, Higher expansion rate of the spleen white pulp size were recorded in the LBT group. The antigen preparation supplemented with adjuvants had positive effects on spleen white pulp expansion following live Leishmania major challenging, nevertheless, based on previous experiment, $100-200 \mu \mathrm{g}$ of LT group could induces satisfactory DTH response which favors leishmania organisms eradication, and higher survival rate after challenging with the live Leishmania major organisms in spite of induction of lower spleen white pulp size expansion.

Keywords: Leishmania major antigen; Challenge; Spleen

\section{Introduction}

Leishmania parasites are vector-borne protozoal pathogens found in the tropical and subtropical regions of both the old and New World. These parasites can cause visceral or cutaneous leishmaniasis which is estimated to be responsible for 500,000 and 1,500,000 new human infections, respectively. Untreated visceral leishmaniasis is often fatal, and there is a significant amount of morbidity associated with the cutaneous disease [1]. Leishmania parasites live in the macrophages as round, non-motile 3-5 $\mu \mathrm{m}$ amastigotes. The sand fly ingests the infected macrophages during the blood meal and the amastigotes are released into stomach of insect. Almost immediately the amastigotes transform into the motile, $10-20 \mu \mathrm{m}$ elongated flagelate promastigote form [2]. Upon intradermal /sub-cutaneous injection with L. major, resistance animals develop a small lesion that is limited and spontaneously heals within 6-8 weeks. By contrast Balb/c mice are highly susceptible to the infection with these organisms [3]. These mice fail to control the infection and develop extensive lesions that parasites metastases to the internal visceral organs primarily to the liver, then spleen, and at last bone marrow, is an event that may lead to the animal's death. Many different genes spanning the mouse genome have been implicated in the susceptibility/resistance to Leishmania. Most of these genes do not map within the MHC systems of either humans or mice, including the SCL-1 gene implicated in the high susceptibility of Balb/c mice to infection with L. major [4]. Lymph node cells of Balb/c mice chronically infected with $L$. major, upon stimulation with the Ldp23 antigen produce high levels of IFN- $\gamma$ and undetectable amounts of IL-4, a typical Th1 response [5]. Sand fly saliva selectively inhibits parasite killing by macrophages and could suppress nitric oxide production [6]. The resistance to leishmania conferred by T-helper type-1 (Th1) cells while the susceptibility is conferred by Th2 cells. Th1 cells secret IL12 and IFN- $\gamma$ where as Th2 cells secret IL- 4, IL-5 and IL-10. It has been shown that IFN- $\gamma$ activates macrophages to express iNOS2, the enzyme catalyzing the formation of nitric oxide; nitric oxide kills the intracellular amastigotes. In contrast, Th2 immune response limits the action of Th1 functions via IL-10 and IL-4, which deactivate macrophages helping intracellular parasite growth and disease progression [7]. Many vaccine strategies have been pursued, including the use of whole cell lysate, killed the avirulent or irradiated parasites. Additionally, DNA vaccines and purified or recombinant parasite antigens have also 
been tested. Most of these strategies have shown some degree of effectiveness in animal models but little or no protection in humans. There is now a general consensus among Leishmania vaccine researchers that parasite persistence may be important for effective protective response and could be achieved by the live attenuated parasite immunization [8]. Although there is no vaccine against cutaneous leishmaiasis to date, several of the vaccine preparations are at advanced stages of clinical testing. In the previous reports of author upon the present preliminarily studied candidate vaccine on two types of mice (susceptible and resistance), results of the DTH responses and the spleen white pulp size expansion rates were satisfactory. An increase in Th1 cytokines (IL-12, IFN- $\gamma$ ) and a reduction in Th2 cytokines (IL4, IL10) observed in the recent experiments of the author (unpublished) could indicate beneficial effects of the new formulated Leishmania antigen. The author for the first time used the alcoholic extract of Teucrium polium as an adjuvant to potentiate immune responses to the new preparation of $L$. major antigen on Balb/c mice with the satisfactory results. T. polium plant is an herb native to the Mediterranian region and the Middle East. Traditionally the herb is used as a remedy in various disease conditions including bacterial and inflammatory diseases. To continue our assessment, we formulated a new Leishmania major cocktail crude antigen as a provisional vaccine and decided to evaluate the effects of this new antigen preparation of Leishmania antigen on Balb/c mice following challenging with live Leishmania promastigotes using the same methodology as we had done in our previous studies including antigen preparation an standardization [9-12].

\section{Materials and Methods}

One hundred and twenty young adult female and male Balb/c mice were obtained from Razi Vaccine and Serum Research Institute and randomly assigned to four standard polycarbonate boxes and they were kept and fed under standard condition and four treatment groups. All groups were fed ad lib with the commercial mice chaw and kept in a well ventilated animal room located in the University of Medical Sciences of Tehran, School of Medicine, Tehran, and Islamic Republic of Iran. The experimental design consisted of three antigen injected groups (LT, LB, LBT) and a control group which received no antigen injection. For detail of the antigen and adjuvant preparations, standardization of the Leishmania antigen preparation including formulation, safety and sterility of the preparations, determination of optimum dosages of antigen for Balb/c mice, please visit the authors previous publications [9-12]. The new formulated antigen combined with adjuvant BCG at levels of $2 \times 10^{5}$ CFU live BCG $/ 0.1 \mathrm{ml}$ or 400 $\mu \mathrm{g} / 0.1 \mathrm{ml}$ alcoholic extract of Teucrium polium [13] or an admixture of BCG and T. polium. Group LT received 100-200 $\mu \mathrm{g} / 0.1 \mathrm{ml}$ of the crude cocktail antigen preparation plus alcoholic extract of Teucrium polium as adjuvant, GroupLB received 100-200 $\mu \mathrm{g} / 0.1 \mathrm{ml}$ [14] of the crude cocktail antigen preparation plus BCG as adjuvant, group LBT received $100-200 \mu \mathrm{g} / 0.1 \mathrm{ml}$ of the crude cocktail antigen preparation plus alcoholic extract of Teucrium polium and BCG as adjuvants. Groups LT, LB and LBT were injected subcutaneously with the antigen preparations at the base of the tail and they were boosted two times using the same doses with a week interval. A week after the last booster, all animals including the control (or normal) group were challenged with $3 \times 10^{5} / 0.1 \mathrm{ml}$ live L. major promastigotes. The protective response to the antigen was observed based on cutaneous lesion induction and survival rate during 70 days from the beginning of the challenge. The protective response to the antigen was observed based on cutaneous lesion induction and rate of the mortality. The animals survived post challenging were euthanized using diethylether, necropsies and spleen was removed and fixed in $10 \%$ buffered formaldehyde solution. The fixed spleen tissues were processed in a tissue processor, paraffin blocks were made and 4-5 microns tissue sections were prepared and stained with Harris Hematoxyline and Eosine method. The expansion rates of the spleen white pulp size was evaluated using a light microscope with eye-piece graticule.

Data obtained from the experiment were analyzed using SPSS (SPSS Inc., Chicago, IL, USA). Means were compared by a standard analysis of variance/ simple factorial tests, and by one and two way, student Newman-Keuls methods. Correlation coefficient analysis was determined on a Pearson average two tailed test of significance.

The study was done in compliance with the Helsinki Declaration, and the protocol was approved by research deputy of Tehran University of Medical Sciences, Tehran, Iran.

\begin{tabular}{|l|l|l|l|l|l|l|}
\hline Groups & Sex & Mean & $\mathbf{N}$ & $\begin{array}{l}\text { Std.Error of } \\
\text { Mean }\end{array}$ & Minimum & Maximum \\
\hline \multirow{2}{*}{ LT } & Male & $5.1185 E 2$ & 6 & 49.49877 & 308.00 & 657.50 \\
\cline { 2 - 7 } & Female & $5.7302 \mathrm{E} 2$ & 3 & 122.55497 & 392.70 & 806.96 \\
\cline { 2 - 7 } & Total & $5.3224 \mathrm{E} 2$ & 9 & 48.74903 & 308.00 & 806.96 \\
\hline \multirow{2}{*}{ LB } & Male & $5.8800 \mathrm{E} 2$ & 1 & - & 588.00 & 588.00 \\
\cline { 2 - 7 } & Female & $6.2052 \mathrm{E} 2$ & 4 & 28.44757 & 565.72 & 699.20 \\
\cline { 2 - 7 } & Total & $6.1401 \mathrm{E} 2$ & 5 & 22.97494 & 565.72 & 699.20 \\
\hline \multirow{2}{*}{ LBT } & Female & $9.1794 \mathrm{E} 2$ & 6 & 114.28406 & 623.40 & 1386.10 \\
\hline \multirow{2}{*}{ Control } & Total & $9.1794 \mathrm{E} 2$ & 6 & 114.28406 & 623.40 & 1386.10 \\
\hline & Male & $4.6177 \mathrm{E} 2$ & 3 & 6.36562 & 451.20 & 473.20 \\
\hline \multirow{2}{*}{ Total } & Male & $5.0444 \mathrm{E} 2$ & 10 & 31.00569 & 308.00 & 657.50 \\
\hline & Female & $7.2918 \mathrm{E} 2$ & 15 & 63.95588 & 392.70 & 1386.10 \\
\hline & Total & $6.3928 \mathrm{E} 2$ & 25 & 45.61674 & 308.00 & 1386.10 \\
\hline
\end{tabular}

Table 1: The results of analysis variance show a significant differences in white pulp size between male and female Balb/c mice $(\mathrm{P}<0.001)$.

\section{Results}

The results of the experiment are appeared in the Table 1 and Figure 1. As can be noticed from the Table 1, the differences between the LT, LB, LBT and control group were different statistically $(\mathrm{P}=0$. 001). Compared to control group, the spleen white pulp size increased in the groups LBT and LB but not in LT group. The higher expansion rate of the spleen white pulp size was found in the LBT group that received the crude cocktail antigen preparation plus BCG and alcoholic extract of $T$. polium as adjuvants; statistically the differences were significant. In this regard, the p values for LT, LB, and control groups were $0.02,0.036$ and 0.005 respectively. Among the groups LT, LB and LBT, the lower spleen white pulp size was seen in the LT group which received the crude cocktail antigen preparation supplemented with alcoholic extract of $T$. polium as adjuvant. In this regard, there was a significant difference between LBT and LT groups $(\mathrm{P}<0.002)$ 
and the differences were significant as well when LBT was compared to the groups of LB (0.03) and control (0.05). It seems that, when BCG and alcoholic extract of T. polium are used together, they show a remarkable synergistic effects (Table 1). Comparing to control group, the crude cocktail antigen supplemented with alcoholic extract of $T$. polium had a negligible effect on spleen white pulp size expansion rate. Spleen is the most important organ of the secondary lymphoid tissue which is structurally composed of white pulp (periarterioral lymphoid tissue with primary or secondary lymphoid follicles) and red pulp (sinuses and blood vessels). The white pulp is where the immune response is induced. The white pulp could react to antigen administration via the expansion of lymphoid constituents including B cell and $\mathrm{T}$ cell dependant areas, results in antibody production. The SWPS expansion rates differed in the female and male LT, LB and LBT and control groups. The largest white pulp size were seen in the female $\mathrm{Balb} / \mathrm{c} \mathrm{LBT}$ group, and smallest white pulp size were seen in the male Balb/c LT group (Table 1). The data obtain from the experiment indicate that comparing to control group, the higher survival rate was seen in LT group which received the antigen preparation plus the alcoholic extract of $T$. polium followed by live $L$. major challenge. The protection rates 70 days post challenging in the LT, LB, LBT and control groups were $50.0 \%, 25.0 \%, 30.0 \%$ and $20.0 \%$ respectively. Comparing, four groups of LT, LB, LBT and control, the lower survival rate was seen in control group challenged by live L. major promastigotes.

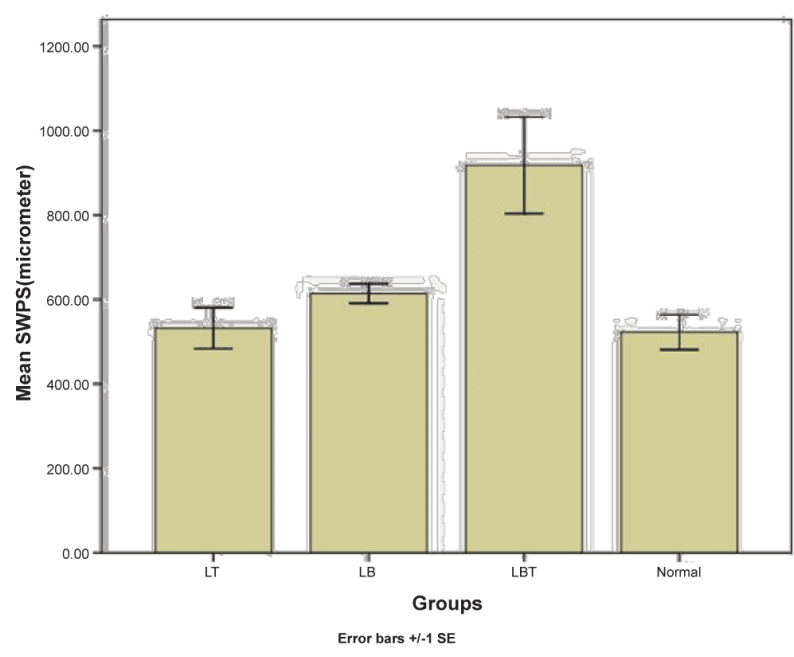

Figure 1: The effects of the crude cocktail $L$. major antigen preparation on spleen white pulp size in the groups LT, LB and LBT challenged with the live $L$. major ptomastigotes, compared to control group. Differences between LBT groups and three groups of LT, LB and control groups is significant statistically $(\mathrm{P}<0.05)$, but differences between three groups of LT, LB and control group is not significant $(\mathrm{p}>0.05)$.

\section{Discussion}

Leishmaniasis is a parasitic infection by an obligate, intracellular protozoan of the genus Leishmania. Localized, cutaneous leishmaniasisis characterized by one or more ulcers with limited selfhealing. The parasite has a developmental stage in mammals, which is mostly intracellular [15]. Using defined vaccination protocols is an interesting unorthodox alternative approach to the discovery of a Leishmania vaccine. Paradoxically with respect to the conventional Th1/Th2 paradigm, many investigators have observed that several leishmanial antigens against which a Th1 response is developed during the infection are not necessarily protective antigens [4,5]. Recent experiments using the virulent mutant phosphoglycan-deficient $L$. major lpg-2 demonstrated that infection of mice with this parasite results in minor disease and long survival of the mutant in the animals' tissue. The protection induced by $\operatorname{lpg} 2$ parasites is basically not associated with enhanced INF- $\gamma$ production in response to leishmanial antigens but clearly with a dramatic suppression of IL- 4 and IL-10 response to the same antigens [3,4]. Killed vaccines have shown limited immunogenicity and efficacy, even when combined with adjuvant either BCG or alum). Interestingly, BCG alone led to a positive leishmanin skin test in some individuals, presumably due to antigenic cross-reactivity between mycobacteria and leishmania organism. Leishmania parasites posses virulence mechanisms that enable the amastigote stage to survive in the hostile environment of the phagolyzosome [16]. Efforts are being made to improve safety of leishmanization by inclusion of drug-sensitive Leishmania mutants containing suicide genes for controlled infection $[17,18]$. The current human vaccines which reviewed related to some experimental studies based on murine models with emphasis on Leishmania major infection relevant to vaccine development. In a human study in Venezuela, a clinical healing rate of more than $95 \%$ was achieved following vaccine administration, and cure was associated with a Th1like immune response invaccinated patients $[19,20]$. Another modified form of vaccine using pasteurized $L$. braziliensis promastigotes and live BCG was effective in the treatment of both refractory mucocutaneous Leishmaniasis and early cases of diffuse cutaneous Leishmaniasis [21]. To date, successful vaccination strategies against Leishmaniasis have been limited to cutaneous Leishmaniasis, with small doses of living virulent $L$. major promastigotes at a selected site, however the results of the vaccination has not been satisfactory [20]. The clinical effectiveness of immunization with the killed vaccine is not acceptable at the present [22]. Phase III clinical trials in Ecuador and Colombia showed that the Leishmania amazonensis vaccine is safe, but not effective $[23,24]$. One study's results show that NKT cells should be considered both when treating active Leishmania infection as well as in the development of vaccines. The reported effects of L.major-activated NKT cells observed in various models of Leishmania infection have been variable and often conflicting which, most of this is probably due to both different infection models and Leishmania strains applied [24]. Recent progress in understanding how the innate immune system recognizes microbial stimuli and regulates adaptive immunity is being applied to vaccine discovery in what is termed "systems vaccinology" [25]. Systems vaccinology is an offshoot of systems biology for which tools of a number of high- throughput technologies including DNA microarrays, RNA-seq., protein arrays, deep sequencing, mass spectrometry along with sophisticated computational tools have been originally developed [26]. The role of IL-10 in promoting pathology of VL has long been demonstrated in human studies. It must be noted that in several of the heat killed Leishmania vaccines, BCG was a common adjuvant and the immune reaction caused by BCG compounded the LST-based interpretation significantly. A meta- analysis further confirmed that LST conversion maybe associated with an immune response that can provide some protection by its ability to distinguish as an population of responders to leishmanial antigen or BCG after vaccination even though such response had a huge variability (16-68\% conversion rate) in these studies [27]. Regarding to above researches and based on the previous 
studies of the author, the new formulated crude cocktail preparation of L. major antigen supplemented by BCG alone or BCG plus new recommended alcoholic extract of plant Teucrium polium as an adjuvant showed a satisfactory immune response which could help animal to contain leishmania infection. In the aforementioned experiments conducted by the author, safety, toxicities and immunity (DTH) of the new preparation and protective results accompanied by cytokine assessment after challenging were evaluated in the murine models [10-12].

Higher white pulp size in the LBT group indicates induction of humoral immunity which it could not protect the animals against Leishmania infection or progression. In addition this antigen preparation also could induce cell mediated immunity which it seems to be a protective response as shown in previous studies. Therefore, supplementation of crude cocktail antigen at levels of $2 \times 10^{5} \mathrm{CFU}$ live BCG $/ 0.1 \mathrm{ml}$ and $400 \mu \mathrm{g} / 01 \mathrm{ml}$ of alcoholic extract of Teucrium polium simultaneously could induce cell mediated and humoral immune responses at 100-200 $\mu \mathrm{g}$ of the antigen in the Balb/c mice model. However as far as LT and LBT groups were concerned, in spite of lower expansion rates of spleen white pulp size which indicates lower non protective humoral immunity, they were shown a satisfactory DTH response.

In the authors previous experiments [9-12] the new formulation of crude cocktail of Leishmania major antigen fortified by alcoholic extract of plant Teucrium polium could be recommended as a candidate vaccine, since compared to the LBT an LB groups, the antigen preparation caused lower mortality and a satisfactory DTH response which could be considered as a protective responses to Leismania major infection. With regard to antigen preparation, a mixture of antigens prepared by using different methods including freezing and thawing, autoclaving, heat inactivation, chemical inactivation and combination of chemical inactivation and freezing and thawing method, offer very many leishmania epitopes. It could be expected that some of these epitopes could induce such an immune response which capable to protect the vaccinated subjects. In the other hand, as shown previously, it seems that the pure alcoholic extract of Teucrium polium could be a safe adjuvant as shown in the Balb/c mice. However, since the extract is used parentally, further investigation is required to elucidate the safety of the adjuvant. In contrast, the crude cocktail antigen preparation plus alcoholic extract of Teucrium polium and BCG or plus BCG alone is not recommended for the provisional vaccine in spite of their potency in both cell mediated or humoral immunity induction since the live BCG could produce mycobacterial infection particularly in the immune compromised or immunodefficient subjects. As seen in the Table 1, the gender of the animals affects the spleen white pulp size expansion rates against the antigen preparation, in this regard, the differences between two groups of female and male Balb/c mice were statistically significant. In fact, it could be attributed partly to different types of hormones produced by the female and male animals including estrogenic/progestronic versus androgenic hormones. At the present time, there are unsolved problems and many questions must be answered, nevertheless the rapid progress in the field of vaccine, vaccination and development of safe and effective vaccines against intracellular infectious agents including lieshmania organisms is promising. We expect that the new antigen formula would show the following characteristics; firstly, could induce a reasonable immune response which activate macrophages therefore it enable macrophages to inhibit propagation or destroy intracellular amastigotes at the early stages of infection, secondly, could inhibit transformation of promastigotes organisms to intracellular forms or amstigotes at the site of the parasite entrance, thirdly, to induce a safe and potent immune response which could help clinically infected individuals to eradicate intracellular form of leishmania organisms, fourthly, from our point of view the side effect of the antigen preparation must be negligible or tolerable when it is administrate, since induction of high levels of cytokines including inflammatory cytokines or activation of a large proportion of lymphocytes will have a grave consequences and finally mass production of antigen preparation as a vaccine must be feasible commercially. On the other hand, this preparation is safer than those supplemented with the live BCG as adjuvant since they could produce mycobacterial infection particularly in the immune deficient individuals.

\section{Conclusion}

The results of the present experiment and previous findings were obtained by the author indicate that the crude cocktail antigen preparation at doses of $100-200 \mu \mathrm{g} / 0.1 \mathrm{ml}$ which had alcoholic extract of Teucrium polium as adjuant could induce an acceptable and satisfactory immune response in the $\mathrm{Balb} / \mathrm{c}$ mice. To evaluate the preparation as a provisional vaccine against Leishmania major infection, the author recommends further investigations conducted in other susceptible species including canine species.

\section{References}

1. Vanloubbeeck Y, Jones DE (2004) The immunology of Leishmania infection and the implications for vaccine development. Ann N Y Acad Sci 1026: 267-272.

2. Killick-Kendrick R (1990) The life-cycle of Leishmania in the sandfly with special reference to the form infective to the vertebrate host. Ann Parasitol Hum Comp 65 Suppl 1: 37-42.

3. Blackwell JM (1996) Genetic susceptibility to leishmanial infections: studies in mice and man. Parasitology 112 Suppl: S67-74.

4. Campos-Neto A (2005) What about Th1/Th2 in cutaneous leishmaniasis vaccine discovery? Braz J Med Biol Res 38: 979-984.

5. Campos -Neto, Soong A, Cordova L, Sant'angelo JL, Skeiky D, et al. (1995) Clonoing and expression of a leishmania donovani gene instructed by a peptide isolated from major histocompatibility complx class II molecules of infected macrophages. J Exp Med 182: 1423-1433.

6. Guevara P, Pinto-Santíni D, Rojas A, Crisante G, Añez N, et al. (2001) Green fluorescent protein-tagged Leishmania in phlebotomine sand flies. J Med Entomol 38: 39-43.

7. Hall LR, Titus RG (1995) Sand fly vector saliva selectively modulates macrophage functions that inhibit killing of Leishmania major and nitric oxide production. J Immunol 155: 3501-3506.

8. Awasthi A, Mathur RK, Saha B (2004) Immune response to Leishmania infection. Indian J Med Res 119: 238-258.

9. Latifynia A, Khamisipour A, Gharagozlou MJ, Bokaie S, Vodjgani M, et al. (2013) Post challenging serum cytokine profile (Th1 \& Th2) in the vaccinated mice $(\mathrm{Balb} / \mathrm{C})$ with a new formulation of Leishmania major antigen. Turkiye Parazitol Derg 37: 233-240.

10. Latifynia A, Mohaghegh Hazrati S (2008) Safety and Toxicity of a New Formulated Leishmania major Preliminary Vaccine in Animal Model Balb/c and Small White Conventional Laboratory Mice. Turkiye Parazitol Derg 32: 103-108.

11. Latifynia A, Hazrati SM (2010) Evaluation of the effects of a new formulation of Leishmania major antigen in Balb/C and conventional white laboratory mice. J Microbiol Immunol Infect 43: 138-146.

12. Latifynia A, Mohaghegh Hazrati S, Mahmodi , Mohebali M (2009) Study on immunity of Leishmania major new crude antigen as a vaccine against leishmaniasis in out bred (resistant) and Balb/c (sensitive) mice. Archives of Razi Institute 64: 27-37. 
Citation: Latifynia A, Gharagozlou M, Khamesipour A, Mohammadi MA, Khansary N (2014) Primary Effects of New Leishmania major Antigen on Balb/c Mice Spleen. J Vaccines Vaccin 5: 253. doi:10.4172/2157-7560.1000253

Page 5 of 5

13. Shahraki MR, Arab MR, Mirimokaddam E, Palan MJ (2007) The effect of Teucrium polium (Calpoureh) on liver function, serum lipids and glucose in diabetic male rats. Iran Biomed J 11: 65-68.

14. Lowry OH, Rosebrough NJ, Farr AL, Randall RJ (1951) Protein measurement with the Folin phenol reagent. J Biol Chem 193: 265-275.

15. Selvapandiyan A1, Duncan R, Debrabant A, Lee N, Sreenivas G, et al. (2006) Genetically modified live attenuated parasites as vaccines for leishmaniasis. Indian J Med Res 123: 455-466.

16. Roberts MT (2006) Current understandings on the immunology of leishmaniasis and recent developments in prevention and treatment. $\mathrm{Br}$ Med Bull 75-76: 115-30.

17. Muyombwe A, Olivier M, Ouellette M, Papadopoulou B (1997) Selective killing of Leishmania amastigotes expressing a thymidine kinase suicide gene. Exp Parasitol 85: 35-42.

18. Davoudi N, Tate CA, Warburton C, Murray A, Mahboudi F, et al. (2005) Development of a recombinant Leishmania major strain sensitive to ganciclovir and 5-fluorocytosine for use as a live vaccine challenge in clinical trials. Vaccine 23: 1170-1177.

19. Cabrera M , Blackwell J M ,Castes M , Trujillo D ,Convit J, et al. (2000) Immunotherapy with live BCG plus heat killed leishmania induces a $\mathrm{T}$ helper 1 -like response in American cutaneous Leishmaniasis patients. Parasite Immunol 22: 73-79.

20. Convit J, Ulrich M, Zerpa O, Borges R, Aranzazu N, et al. (2003) Immunotherapy of american cutaneous leishmaniasis in Venezuela during the period 1990-99. Trans R Soc Trop Med Hyg 97: 469-472.
21. Ahmed SB, Bahloul C, Robbana C, Askri S, Dellagi K (2004) A comparative evaluation of different DNA vaccine candidates against experimental murine leishmaniasis due to L.major. Vaccine 22: 1631-1639.

22. Armijos RX, Weigel MM, Calvopina M, Hidalgo A, Cevallos W, et al. (2004) Safety, Immunogenicity, and efficacy of an autoclaved Leishmania amazonensis vaccine plus BCG adjuvant against New World cutaneous Leishmaniasis. Vaccine $22: 1320-1326$.

23. Velez ID , Gilchrist K, Arbelaez MP , Rojas CA, Puerta JA, et al. (2005) Failure of a killed leishmania amazonensis vaccine against American cutaneous Leishmaniasis in Colombia . Trans Royal Soc Trop Med Hyg 99 : 593-598.

24. Griewank KG, Lorenz B, Fischer MR, Boon L, Lopez Kostka S, et al (2014) Immune modulating effects of NKT cells in a physiologically low dose Leishmania major infection model after aGalCer analog PBS57 stimulation. PLoS Negl Trop Dis 8: e2917.

25. Gannavaram S, Dey R, Avishek K, Selvapandiyan A, Salotra P, et al. (2014) Biomarkers of safety and immune protection for genetically modified live attenuated leishmania vaccines against visceral leishmaniasis - discovery and implications. Front Immunol 5: 241.

26. Pulendran B, Li S, Nakaya HI (2010) Systems vaccinology. Immunity 33: 516-529.

27. Noazin S, Khamesipour A, Moulton LH, Tanner M, Nasseri K, et al. (2009) Efficacy of killed whole-parasite vaccines in the prevention of leishmaniasis: a meta-analysis. Vaccine 27: 4747-4753. 